\title{
Safety and efficacy of vernakalant for acute cardioversion of atrial fibrillation: an update
}

This article was published in the following Dove Press journal:

Vascular Health and Risk Management

22 April 2013

Number of times this article has been viewed

\author{
Yukiomi Tsuji \\ Dobromir Dobrev'-3 \\ 'Institute of Pharmacology, Faculty \\ of Medicine, University Duisburg- \\ Essen, Essen, ${ }^{2}$ Division of \\ Experimental Cardiology, Medical \\ Faculty Mannheim, Heidelberg \\ University, Mannheim, ${ }^{3}$ Deutsches \\ Zentrum für Herz-Kreislauf- \\ Forshcung [German Center \\ for Cardiovascular Research], \\ partner site Heidelberg/Mannheim, \\ Mannheim, Germany
}

\begin{abstract}
Intravenous vernakalant has recently been approved in Europe as an atrial-selective antiarrhythmic drug for the conversion of recent-onset atrial fibrillation (AF). It inhibits atrialselective $\mathrm{K}^{+}$currents $\left(\mathrm{I}_{\mathrm{K}, \mathrm{ACh}}\right.$ and $\left.\mathrm{I}_{\mathrm{Kur}}\right)$ and causes rate-dependent atrial-predominant $\mathrm{Na}^{+}$channel block, with only a small inhibitory effect on the rapid delayed rectifier $\mathrm{K}^{+}$current $\left(\mathrm{I}_{\mathrm{Kr}}\right)$ in the ventricle. Due to its atrial-selective properties, vernakalant prolongs the effective refractory period of the atria with minimal effects on the ventricles, being associated with a low proarrhythmic risk for torsades de pointes arrhythmias. Five pivotal clinical trials consistently demonstrated that vernakalant rapidly terminates AF with stable maintenance of sinus rhythm for up to 24 hours. A head-to-head comparative trial showed that the 90-minute conversion rate of vernakalant was substantially higher than that of amiodarone. Initially, a longer-acting oral formulation of vernakalant was shown to be effective and safe in preventing AF recurrence after cardioversion in a Phase IIb study. However, the clinical studies testing oral vernakalant for maintenance of sinus rhythm after AF cardioversion were prematurely halted for undisclosed reasons. This review article provides an update on the safety and efficacy of intravenous vernakalant for the rapid cardioversion of AF.
\end{abstract}

Keywords: atrial fibrillation, antiarrhythmic drug, atrial-selective $\mathrm{K}^{+}$currents, $\mathrm{Na}^{+}$channel block, ventricles

\section{Introduction}

Atrial fibrillation (AF) is the most common arrhythmia and its prevalence is expected to rise dramatically owing to an aging population. ${ }^{1}$ AF causes symptoms such as palpitations, dizziness, breathlessness, and chest pain, and is associated with cardiovascular morbidity and mortality, mainly due to embolic stroke and heart failure (HF). ${ }^{1}$ Management of AF patients is focused on reducing symptoms and preventing complications associated with the arrhythmia. Two therapeutic approaches are available: a "rhythm control" strategy, terminating AF and maintaining sinus rhythm, and a "rate control" strategy, allowing the patient to remain in AF but controlling the ventricular response. Both strategies require proper anticoagulation to prevent AF-related risks of thromboembolic complications. ${ }^{1}$ Since several large randomized trials could not demonstrate the superiority of a rhythm control approach, ${ }^{2}$ the choice is not imperative. However, restoration and maintenance of sinus rhythm is the most attractive option because of reduction of symptoms and improvement in exercise tolerance and quality of life. ${ }^{2}$

Antiarrhythmic drugs are considered first-line agents for rhythm control. Currently available and commonly used antiarrhythmic drugs are limited by incomplete efficacy
Correspondence: Dobromir Dobrev Institute of Pharmacology, Faculty of Medicine, University Duisburg-Essen, Hufelandstr 55, D-45I 22

Essen, Germany

Tel +4920I 7333477

Email dobromir.dobrev@uk-essen.de 
for achieving and maintaining sinus rhythm and/or by side effects such as life-threatening ventricular proarrhythmias or severe extra-cardiac toxicities. ${ }^{3-5}$ Amiodarone is the most effective drug for rhythm control but AF recurs in a substantial portion of cases. It is often discontinued due to serious systemic side effects such as thyroid and lung dysfunction. ${ }^{3-5}$ Therefore, there is a clear need for safer and more effective pharmacological strategies for rhythm control. ${ }^{6}$

Vernakalant is a compound with atrial-selective ionchannel blocking properties that has recently been introduced for the acute management of AF. ${ }^{6,7}$ Its intravenous formulation has been approved for the conversion of recent-onset AF in Europe and other parts of the world, but not in the USA. Vernakalant inhibits atrial-selective $\mathrm{K}^{+}$currents, including the ultra-rapidly activating delayed rectifier $\mathrm{K}^{+}$current $\left(\mathrm{I}_{\text {Kur }}\right)$ and acetylcholine-activated inward rectifier $\mathrm{K}^{+}$current $\left(\mathrm{I}_{\mathrm{K}, \mathrm{ACh}}\right)$, and causes rate-dependent atrial-preferential $\mathrm{Na}^{+}$channel block, with only a small inhibitory effect on the rapidly activating delayed rectifier $\mathrm{K}^{+}$current $\left(\mathrm{I}_{\mathrm{Kr}}\right)$ in the ventricle. ${ }^{8,9}$ Due to its atrial-selective properties, vernakalant prolongs the effective refractory period (ERP) of the atria with minimal effects on the ventricles, ${ }^{10}$ which explains the low proarrhythmic risk for torsades de pointes (TdP) arrhythmias. ${ }^{6,7}$ Several randomized clinical trials consistently demonstrated the efficacy and safety of intravenous vernakalant for the cardioversion of recentonset AF. ${ }^{11-15}$ A head-to-head comparative trial showed that the 90-minute conversion rate of vernakalant was substantially greater than that of amiodarone. ${ }^{16}$ Although a longer-acting oral formulation of vernakalant showed some efficacy and good safety for prevention of AF recurrence after electrical cardioversion, further development of oral vernakalant was halted, ${ }^{17}$ probably because of insufficient efficacy to maintain sinus rhythm. The lack of an oral formulation for long-term therapy is an obvious limitation for the use of intravenous vernakalant. In addition, one unpublished trial of intravenous vernakalant was suspended following a single unexpected serious adverse event of cardiogenic shock, pending assessment by the US Food and Drug Administration., ${ }^{5,7}$ The aim of this review article is to summarize the putative mechanisms of action of vernakalant and to provide an update on the clinical evidence for efficacy and safety of vernakalant for cardioversion of AF.

\section{Pharmacological actions and therapeutic efficacy of vernakalant Mechanism of action}

Vernakalant is an antiarrhythmic agent that has predominant actions on atrial electrophysiology. A human electrophysiological study demonstrated that vernakalant infusion dose-dependently prolongs atrial ERP but has no significant effect on ventricular ERP or QT interval. ${ }^{10}$ Atrial selectivity, thereby avoiding ventricular proarrhythmia, can be achieved by targeting atrial-selective channels, such as $\mathrm{I}_{\mathrm{Kur}}$ and $\mathrm{I}_{\mathrm{K}, \mathrm{ACh}}$, by atrial-preferential inhibition of excitability through exploiting state-selective $\mathrm{Na}^{+}$channel blocking properties or by high selectivity for rapid rhythms like AF. ${ }^{6}$

Vernakalant blocks several $\mathrm{K}^{+}$channels. It inhibits $\mathrm{I}_{\mathrm{Kur}}$ in the open state, with preserved efficacy at high stimulation frequencies. ${ }^{8}$ The atrial-selective $\mathrm{I}_{\mathrm{K}, \mathrm{ACh}}$ current is potently blocked by vernakalant. ${ }^{18,19}$ Vernakalant also targets Kv4.3 and human ERG (hERG) channels that correspond to the transient outward current $\left(\mathrm{I}_{\mathrm{to}}\right)$ and $\mathrm{I}_{\mathrm{Kr}}$, respectively, although the contribution of $\mathrm{I}_{\text {to }}$ to repolarization is lower in ventricles than in atria. ${ }^{8}$ In contrast, $\mathrm{I}_{\mathrm{Kr}}$ is an important repolarizing current in ventricular cells. Its blockade causes QT interval and action potential duration prolongation, predisposing to $\mathrm{TdP}$ arrhythmias through the development of arrhythmia-triggering early afterdepolarization and/or an increased dispersion of repolarization. ${ }^{6}$ However, the potency of vernakalant in blocking hERG channels is up to 100 -fold lower than that of class IC antiarrhythmic drugs (flecainide or propafenone). ${ }^{8}$ Late $\mathrm{Na}^{+}$current $\left(\mathrm{I}_{\mathrm{Na} \text {,late }}\right)$ inhibition by vernakalant is protective against the proarrhythmia from $\mathrm{I}_{\mathrm{Kr}}$ blockade. ${ }^{20}$ Accordingly, clinical trials showed little changes in QT interval and a very low incidence of ventricular tachyarrhythmia following vernakalant infusion, which was not significantly different from placebo recipients. ${ }^{9}$

Vernakalant causes an open-channel block of $\mathrm{Na}^{+}$channel Nav1.5 $\alpha$-subunits that underlie the atrial $\mathrm{I}_{\mathrm{Na}}{ }^{8,18}$ At physiological heart rates, the block of Nav1.5 channels by vernakalant is weak because of its rapid unbinding kinetics from the channel, ${ }^{8,18}$ which is consistent with the small increase in QRS interval (a marker of ventricular conduction velocity) observed in clinical trials. ${ }^{12-14}$ In addition, the effects of vernakalant on $\mathrm{Na}^{+}$channels are voltage and rate dependent, resulting in an enhanced inhibitory potency at depolarized potentials and rapid rates, like in fibrillating atria. ${ }^{8}$ The resting membrane potential of normal atrial myocytes is $10 \mathrm{mV}$ more depolarized than that of normal ventricular myocytes. When atrial myocytes fail to repolarize fully, as can happen during AF, the atrioventricular difference in resting membrane potential is further accentuated and a large fraction of atrial $\mathrm{Na}^{+}$channels is inactivated. This reduces the $\mathrm{Na}^{+}$channel reserve predominantly in the atria and allows vernakalant to inhibit preferentially atrial $\mathrm{Na}^{+}$channels. ${ }^{8}$ Although such voltage and rate dependency 
is also typical for flecainide and propafenone, they do not show atrial selectively, ${ }^{8,21,22}$ and the important difference is that vernakalant exhibits rapid unbinding kinetics from $\mathrm{Na}^{+}$ channels. ${ }^{8}$ Therefore, $\mathrm{Na}^{+}$channel block with rapid unbinding kinetics has recently been identified as a promising option for atrial-selective drug treatment of $\mathrm{AF}^{23,24}$ Besides vernakalant, the antianginal agent ranolazine and amiodarone have also been shown to cause atrial-selective $\mathrm{Na}^{+}$ channel inhibition. ${ }^{22,25-28}$ A recent ex vivo study of human atrial tissue from sinus rhythm and chronic AF showed that vernakalant causes a rate-dependent reduction in maximum action potential upstroke velocity and that ERP is increased more than action potential duration, resulting in postrepolarization refractoriness. ${ }^{19}$ The ion-channel inhibitory properties and electrophysiological effects of vernakalant are summarized and compared with those of amiodarone and flecainide, the agents most commonly used in AF, in Table 1 . Vernakalant exerts antifibrillatory actions that are shared by amiodarone and flecainide by prolonging atrial ERP and/or reducing atrial excitability.

The myocardial sleeves of pulmonary veins (PVs) are an important source of ectopic activities that initiate and maintain paroxysmal $\mathrm{AF}^{29}$ Several studies have proposed enhanced automaticity, ${ }^{30}$ triggered activity, ${ }^{31,32}$ and localized re-entry in $\mathrm{PVs}^{33}$ as the underlying mechanisms of clinical AF. The pure $\mathrm{Na}^{+}$channel blocker pilsicainide terminated AF by modifying ERP heterogeneity and conduction properties in the PVs and at the PV-left atrial junction in patients. ${ }^{34} \mathrm{~A}$ recent study in canine PV sleeve preparations has shown that vernakalant increases the ERP in a rate-dependent manner and that vernakalant suppresses delayed afterdepolarization-mediated triggered activity induced by burst pacing in the presence of isoprenaline and high $\mathrm{Ca}^{2+} .{ }^{35}$ The authors speculated that vernakalant may cause a reduction in intracellular $\mathrm{Ca}^{2+}$ loading, secondary to the reduction in intracellular $\mathrm{Na}^{+}$loading at rapid rates. ${ }^{35}$ Whether these effects could contribute to the clinical efficacy of vernakalant is unknown.

\section{Clinical efficacy}

The efficacy of intravenous vernakalant for cardioversion of recent-onset AF has been demonstrated in six clinical trials (Table 2). One of these was the first dose-finding Phase II study (Conversion of Rapid Atrial Fibrillation Trial [CRAFT]). In all subsequent studies (Atrial arrhythmia Conversion Trial [ACT] I, II, III, IV, and A phase III superiority study of Vernakalant vs amiodarone in subjects with Recent Onset atrial fibrillation [AVRO]), vernakalant was administered by a 10-minute infusion of $3 \mathrm{mg} / \mathrm{kg}$ followed by a 15 -minute observation period then a second 10-minute infusion of $2 \mathrm{mg} / \mathrm{kg}$ if still in AF. The results of these trials have been reviewed in excellent articles elsewhere. ${ }^{7,36-41}$ Based on these data, intravenous vernakalant was approved by the European Commission for the rapid cardioversion of recent-onset $\mathrm{AF}$ (lasting $\leq 7$ days for non-surgery patients and $\leq 3$ days for post-cardiac-surgery patients) to sinus rhythm in adults. ${ }^{9}$ Following, we update the currently available efficacy and safety information obtained in clinical trials and discuss the potential consequences for the clinical use of vernakalant.

The ACT I ${ }^{12}$ and $\mathrm{III}^{14}$ tested vernakalant in patients with short-duration AF (3 hours to 7 days) and long-duration AF ( 8 to 45 days). The primary endpoint in both studies was the proportion of patients with short-duration AF who had treatment-induced conversion of AF to sinus rhythm for at least 1 minute within 90 minutes of treatment infusion. The secondary endpoint in short-duration AF patients included the time to conversion from the first administration and the proportion of patients who remained in sinus rhythm at 24 hours. Another secondary endpoint was conversion of AF in patients with longer-duration AF.

Vernakalant effectively and rapidly converted AF to sinus rhythm compared with placebo. The primary endpoint was

Table I lon-channel blockade properties and electrophysiological effects

\begin{tabular}{|c|c|c|c|c|c|c|c|c|c|c|}
\hline & \multicolumn{6}{|c|}{ lon-channel blocking } & \multirow[t]{2}{*}{ References } & \multicolumn{3}{|c|}{$\begin{array}{l}\text { Mechanisms of recent-onset } \\
\text { AF conversion }\end{array}$} \\
\hline & $\begin{array}{l}\mathrm{I}_{\mathrm{Na}} \text { (atrial } \\
\text { selectivity) }\end{array}$ & $I_{\text {Kur }}$ & $I_{K, A C h}$ & $I_{\text {to }}$ & $I_{\mathrm{Kr}}$ & $I_{\mathrm{Na}, \text { late }}$ & & ERP & Excitability & $\begin{array}{l}\text { Ectopic } \\
\text { activity }\end{array}$ \\
\hline Vernakalant & $\bullet(+)$ & $\bullet$ & $\bullet$ & $\bullet$ & $\bullet$ & $\bullet$ & $\begin{array}{l}\text { Fedida et al, }{ }^{8} \text { Fedida, }{ }^{18} \\
\text { Orth et al }{ }^{20}\end{array}$ & $\uparrow$ & $\downarrow$ & $\downarrow ?$ \\
\hline Amiodarone & $\bullet(+)$ & $\bullet$ & $\bullet$ & $\bullet$ & $\bullet$ & - & $\begin{array}{l}\text { Suzuki et al, }{ }^{28} \text { Kodama } \\
\text { et al, }{ }^{56} \text { Gautier et al }{ }^{57}\end{array}$ & $\uparrow$ & $\downarrow$ & $\downarrow ?$ \\
\hline Flecainide & $\bullet(-)$ & - & $\bullet$ & $\bullet$ & $\bullet$ & $\bullet$ & $\begin{array}{l}\text { Burashnikov and } \\
\text { Antzelevitch, }{ }^{22} \text { Aliot } \\
\text { et al, }{ }^{58} \text { Voigt et al }{ }^{59}\end{array}$ & $\uparrow$ & $\downarrow$ & $\downarrow ?$ \\
\hline
\end{tabular}

Notes: The - denotes action of agents on ion channel. It is unknown whether an inhibitory effect on ectopic activity can contribute to the clinical efficacy of agents.

Abbreviations: AF, atrial fibrillation; ERP, effective refractory period; $I_{K u r}$, ultra-rapidly activating delayed rectifier $\mathrm{K}^{+}$current; $I_{K, A C h}$, acetylcholine-activated inward rectifier $\mathrm{K}^{+}$current; $\mathrm{I}_{\mathrm{Kr}}$, rapidly activating delayed rectifier $\mathrm{K}^{+}$current; $\mathrm{I}_{\mathrm{Na}}, \mathrm{Na}^{+}$current; $\mathrm{I}_{\mathrm{Na}, \mathrm{late}}$, late $\mathrm{Na}^{+}$current; $\mathrm{I}_{\mathrm{to}}$, transient outward current. 


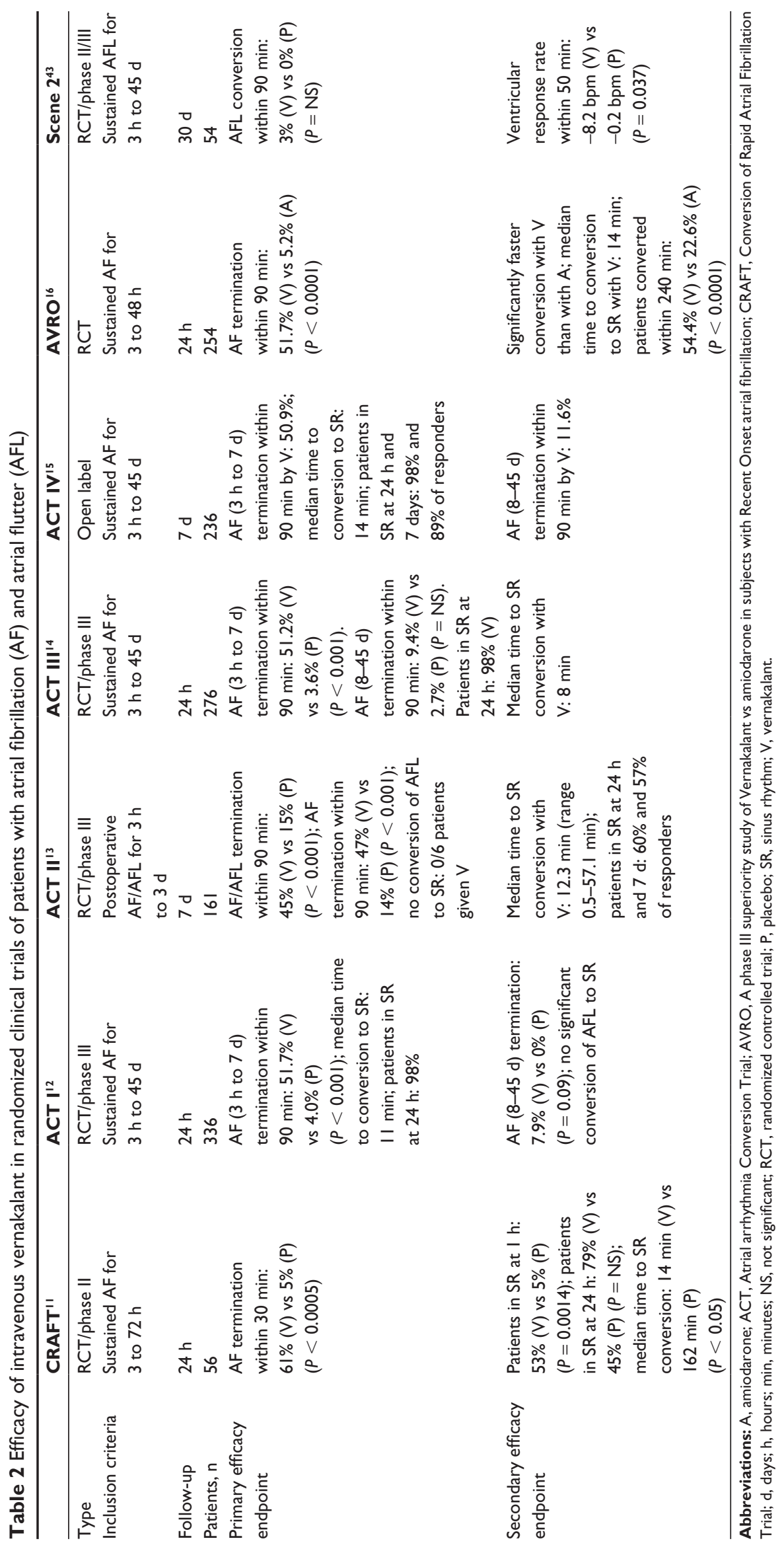


achieved in $51.7 \%$ of vernakalant-treated patients versus $4.0 \%$ of placebo (in ACT $\mathrm{I}^{12}$ ) and in $51.2 \%$ versus $3.6 \%$ (in ACT III ${ }^{14}$ ). Responders given vernakalant showed rapid conversion (median time of 11 and 8 minutes, in ACT I and III, respectively) and a low rate of AF relapse within 24 hours $\left(1.3 \%\right.$ and $2.3 \%$ in ACT I and III, respectively). ${ }^{12,14}$

The duration of AF affected the efficacy of vernakalant. Patients with AF lasting 3 to 48 hours given vernakalant demonstrated the highest conversion rate of $62.1 \%$ (versus $4.9 \%$ for placebo). Conversion rate in patients with AF lasting 3 to 7 days decreased to $23.8 \%$ but remained significant (versus $0 \%$ for placebo, in ACT I). However, longer-duration AF rarely responded favorably, and the conversion rate was not statistically different from placebo (7.9\% vs $0 \%$ and $9 \%$ vs 3\% in ACT I and III, respectively). ${ }^{12,14}$ Similar results were obtained in the open-label ACT IV study. ${ }^{15}$

The ACT II trial included patients with AF lasting 3 to 72 hours that occurred between 24 hours and 7 days after coronary artery bypass graft and/or valvular surgery. ${ }^{13}$ The primary endpoint was the conversion to sinus rhythm within 90 minutes of dosing, which was met in $47 \%$ of vernakalanttreated patients compared with $14 \%$ of placebo-treated patients. The median time to conversion was 12 minutes.

The active-controlled AVRO trial compared the efficacy of vernakalant with amiodarone (a 1 hour infusion of $5 \mathrm{mg} / \mathrm{kg}$ followed by a maintenance infusion of $50 \mathrm{mg}$ over 1 additional hour) in recent-onset AF (of 3 to 48 hours duration) ${ }^{16}$ The primary endpoint was the proportion of patients who achieved sinus rhythm at 90 minutes after initiating infusion. Treatment with vernakalant converted $51.7 \%$ of patients to sinus rhythm at 90 minutes compared with $5.2 \%$ of patients treated with amiodarone. The proportion of vernakalant- or amiodarone-treated patients converted to sinus rhythm within 240 minutes was $54.4 \%$ and $22.6 \%$, respectively. These results are attributed to a slow onset of action of amiodarone and do not indicate that vernakalant is superior to amiodarone in treating recent-onset AF.

These pivotal Phase III trials included patients with a history of hypertension, mild to moderate HF (except New York Heart Association functional class IV), myocardial infarction, ischemic heart disease, and valvular heart disease. There were no significant differences in response to vernakalant injection in any subgroup. However, there was a trend toward decreased efficacy in patients with a history of $\mathrm{HF}(26.9 \% \mathrm{vs}$ $50.0 \%$ for no HF history). ${ }^{9}$ A sub-analysis of patients with a history of ischemic heart disease showed that the rate of AF conversion by vernakalant was not influenced by the presence of ischemic heart disease. ${ }^{42}$ Serious adverse events and drug discontinuations due to adverse events were similar in patients with and without ischemic heart disease. No cases of TdP, ventricular fibrillation, or death were recorded in patients with ischemic heart disease. Of note, since patients were excluded in these trials if they had acute myocardial infarction, acute coronary syndrome, or cardiac surgery within 30 days before enrollment (except for the ACT II trial just discussed), the results cannot be extrapolated to patients with acute ischemia.

Vernakalant was ineffective for the treatment of atrial flutter in nonsurgical patients and cardiac surgery patients in the ACT $\mathrm{II}^{13}$ and ACT $\mathrm{III}^{14}$ trials, respectively. Moreover, these studies showed that approximately $10 \%$ of AF patients treated with vernakalant developed atrial flutter in the first 90 minutes, which either restored to sinus rhythm spontaneously or was restored by electrical cardioversion. A recent Phase II/III randomized, double-blind, placebo-controlled trial (Scene 2, Table 2) has confirmed the ineffectiveness of vernakalant in converting atrial flutter. ${ }^{43}$ Vernakalant did not restore sinus rhythm in patients with atrial flutter $(3 \%$ versus $0 \%$ in the placebo group). The treatment increased the atrial flutter cycle length by an average of 55 milliseconds and decreased ventricular response rates by $8.2 \mathrm{bpm}$ from baseline. Importantly, there was no occurrence of 1:1 AV conduction. This is explained by the combined effects of vernakalant on flutter cycle length and AV nodal refractoriness: ${ }^{43}$ besides increasing the atrial flutter cycle length, vernakalant causes a small but significant prolongation of AV nodal refractoriness. ${ }^{10}$ The lack of 1:1 AV conduction appears a clinically important advantage of vernakalant over class IC drugs like flecainide and propafenone that can cause atrial flutter, termed "IC flutter," which is often associated with a 1:1 AV conduction. Although infrequent, this pattern of proarrhythmia has been reported in a patient receiving vernakalant infusion. ${ }^{44}$

Oral vernakalant initially showed promise for maintenance of sinus rhythm in a Phase IIb study. ${ }^{17}$ Patients with symptomatic AF persisting for 72 hours to 6 months were randomized to receive oral vernakalant $(150,300$, or $500 \mathrm{mg})$ or placebo twice daily for up to 90 days. The efficacy analysis was conducted on 605 patients who entered the maintenance phase on day 3 after cardioversion. At day $90,49 \%$ of the $500 \mathrm{mg}$ vernakalant group maintained sinus rhythm versus $36 \%$ of the placebo group $(P=0.021)$. The median time to the first recurrence of AF was $>90$ days in the vernakalant group versus 29 days with placebo. The drug was well tolerated during exposure for up to 90 days, and no proarrhythmic reactions were observed. Vernakalant was not effective at the lower doses. 


\section{Side effects and safety}

Clinical trials have consistently demonstrated that vernakalant is a well-tolerated and safe antiarrhythmic agent. Due to the short half-life of vernakalant ( $\sim 2$ hours), safety evaluations have focused mainly on adverse events within the first 24 hours of administration. The most common adverse events are dysgeusia (19\%), sneezing (13\%), paresthesia (7\%), and nausea (7\%), ${ }^{9}$ which are likely to be related to $\mathrm{Na}^{+}$channel blockade in the central nervous system. These side effects have been considered mild or moderate and not treatment limiting. Related serious adverse events are mostly of cardiovascular origin and include hypotension, bradycardia, and ventricular arrhythmia. ${ }^{9}$ The data obtained from clinical trials are summarized in Table 3 .

\section{Electrocardiogram parameters and risk of ventricular arrhythmias}

An in vivo human electrophysiology study and the CRAFT trial did not find a significant change in QRS or heart rate-corrected QT interval (QTc) by the infusion of vernakalant. ${ }^{10,11}$ In contrast, pivotal trials (ACT I, II, and III) showed that vernakalant increases the QRS and QTc intervals between 5 minutes and 2 hours after the start of infusion. ${ }^{12-14}$ This finding suggests that the atrial selectivity of vernakalant is not absolute and that the drug carries a proarrhythmic risk. Overall, no TdP has been documented within 24 hours of vernakalant administration, except for one case in which the class III antiarrhythmic drug ibutilide was given on top of vernakalant (Scene 2). ${ }^{43}$ Two patients experienced TdP episodes 32 hours and 16-17 days, respectively, after vernakalant administration (ACT I). ${ }^{12} \mathrm{QT}$ interval prolongation led to drug discontinuation in one case only (ACT I). ${ }^{12}$ The incidence of non-sustained ventricular tachycardias reported in vernakalant-treated patients appears comparable to that in placebo-treated patients. However, some cases had drug discontinuation because of ventricular bigeminy (ACT I), ${ }^{12}$ monomorphic sustained (ACT III) ${ }^{14}$ and nonsustained VT (AVRO) ${ }^{16}$ during the first 2 hours. In addition, ventricular arrhythmia events occurred more frequently in patients with a history of HF. ${ }^{9}$ Ventricular arrhythmias unrelated to TdP have been identified as an adverse event of special significance. ${ }^{9}$

\section{Serious adverse events}

There was one case of death considered related to vernakalant and 26 cases $(2.9 \%)$ of serious adverse events within the first 24 hours out of 889 vernakalant-treated patients in clinical trials. Hypotension was the most frequent vernakalant-related serious adverse event (eight patients, 1.0\%). Others include bradycardia (seven patients), complete AV block (two patients), and cardiogenic shock (one patient). One death that occurred in the ACT III study was a 64-year-old patient with a history of severe aortic stenosis and New York Heart Association class II HF. At the time of admission, he was suffering from acute coronary syndrome and AF with a ventricular rate of $\sim 150 \mathrm{bpm}$. The intravenous metoprolol used for ventricular rate control caused transient hypotension. Despite the hemodynamic instability, the patient received the two infusions of vernakalant and became hypotensive after each infusion. Subsequently, the patient developed ventricular fibrillation and resuscitation was unsuccessful. ${ }^{14}$

Hypotensive episodes usually occur within 15 minutes of following vernakalant infusion, are transient, and generally resolve without pharmacological intervention. However, significant hypotension that required treatment with intravenous fluids and/or norepinephrine was reported in four cases (ACT I, II, and III trials), two of which had HF. ${ }^{12-14}$

Bradycardia events are primarily associated with $\mathrm{AF}$ termination. A clinically significant bradycardia that lasted $\leq 20$ minutes from the time of conversion of AF was documented in four patients (ACT IV). ${ }^{15}$ Complete AV block occurred in a 90 -year-old woman (ACT I) ${ }^{12}$ and a cardiac surgery patient (ACT II) ${ }^{13}$ In the former case, it occurred after electrical cardioversion, which was performed $\approx 2.5$ hours after the second infusion and, in the latter case, during the first infusion of vernakalant. Cardiogenic shock occurred in a patient who was diagnosed later as having tachycardiainduced cardiomyopathy (ACT I). ${ }^{12}$ The patient had hypotension after the first infusion, which responded to a saline intravenous bolus injection, and did not receive the second infusion. He underwent electrical cardioversion a few hours later then developed cardiogenic shock.

Deaths occurred up to 30 days after study drug infusion in five vernakalant patients (ACT I, ACT IV, and AVRO), but none was considered related to this medication. ${ }^{12,15,16}$

\section{Clinical considerations}

The conventional class IC (flecainide, propafenone) and class III (amiodarone and ibutilide) antiarrhythmic drugs that are commonly used for the acute termination of recentonset AF have shown limited efficacy and raised substantial safety concerns. ${ }^{3-5}$ In particular, amiodarone's onset of action is extremely slow, lasting up to 24 hours; flecainide and propafenone cannot be given when HF, coronary artery disease, or acute ischemia are present; and ibutilide carries a high risk of causing life-threatening TdP arrhythmias, 


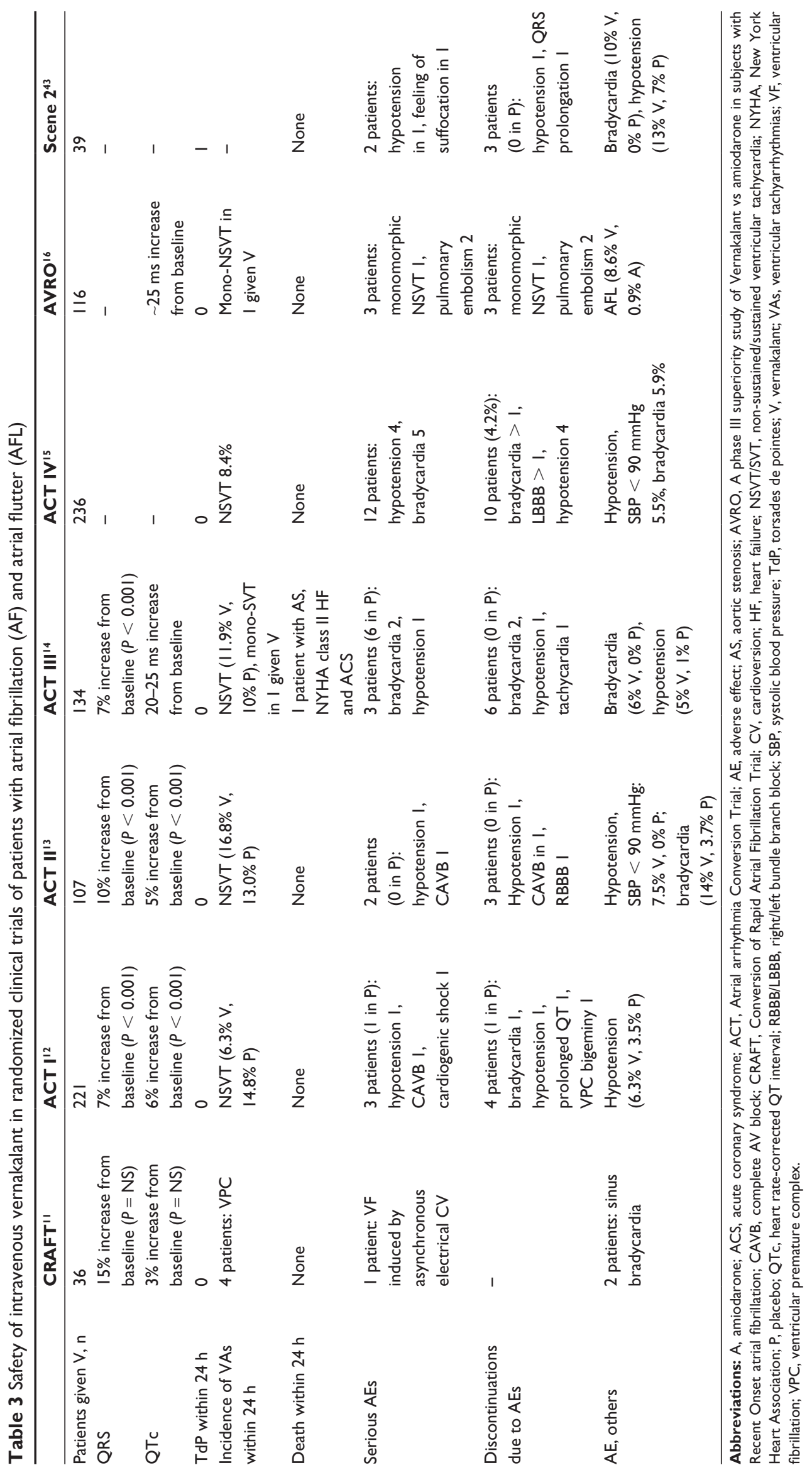


especially in patients with HF. Based on data from the six randomized clinical trials, intravenous vernakalant has a strong advantage over the traditional competitors because of its prompt onset of action, atrial selectivity, and low risk of TdP proarrhythmia. Moreover, vernakalant can be used for a broad range of patients with structural heart diseases and is not contraindicated in patients with stable coronary heart disease, hypertensive heart disease, or $\mathrm{HF}^{45}$ However, with the exception of the AVRO study, clinical trials comparing the efficacy of intravenous vernakalant with other antiarrhythmic drugs have not been published. Ongoing trials are being carried out for direct comparison with flecainide ${ }^{46}$ and ibutilide. ${ }^{47}$ Recently, a meta-analysis was undertaken using a mixed-treatment comparison method to assess the relative efficacy of intravenous vernakalant compared with the more commonly used antiarrhythmic drugs in eliciting cardioversion within 2 hours among patients with short-duration AF ( $<48$ hours). ${ }^{48}$ Vernakalant (intravenous), flecainide (intravenous and oral), and propafenone (intravenous) were more efficacious in pair-wise comparisons than placebo. Vernakalant (intravenous) was observed to be statistically more effective than propafenone (intravenous). Results on the comparative efficacy of vernakalant (intravenous) versus flecainide (intravenous and oral) were not definitive, potentially due to a lack of power to show a difference between treatments or a lack of comparability between patient samples. ${ }^{48}$ These results suggest vernakalant is an efficient and relatively safe antiarrhythmic drug that can be effectively used for the cardioversion of short-duration AF, regardless of presence of structural heart disease.

Results of clinical trials showed that the efficacy of vernakalant decreases with the duration of AF. The conversion rate was substantially lower in AF lasting 3 to 7 days than 3 to 48 hours. Longer-duration AF ( 8 to 45 days) rarely responded to vernakalant. This is in line with previous findings that showed that the efficacies of cardioversion by conventional antiarrhythmic drugs are diminished along with the duration of $\mathrm{AF}^{49}$ This therapeutic resistance to antiarrhythmic drugs is attributed to the progression and severity of atrial remodeling, possibly involving both electrophysiological and structural changes. ${ }^{50,51}$ These findings, together with the fact that there was a trend toward decreased efficacy of vernakalant in patients with a history of $\mathrm{HF},{ }^{9}$ suggest a lower efficacy of vernakalant to cardiovert AF and maintain sinus rhythm in structurally remodeled atria. The development of oral vernakalant for sinus rhythm maintenance was terminated in 2012, probably because of its low efficacy in maintaining sinus rhythm.
In a recently published systematic review and metaanalysis, vernakalant was not associated with a significant difference in serious adverse events compared with controls. ${ }^{52}$ Nevertheless, its safety remains a matter of concern. Cardiogenic shock occurred in two patients (in ACT $\mathrm{I}^{12}$ and unpublished ACT V), one of whom died. Serious hypotension and clinically significant bradycardia were more frequently documented in vernakalant-treated patients. Two patients had complete AV block - one during vernakalant infusion and the other within hours of vernakalant infusion. ${ }^{12,13}$

Vernakalant-related hypotension can be attributed to its negative inotropic effect, which has been shown in experimental studies, ${ }^{9}$ although the underlying mechanisms have not been directly addressed. The negative inotropic effect of vernakalant observed in anaesthetized dogs occurred only at high plasma concentrations (a three-fold or greater margin above the maximum concentration observed at therapeutic doses). ${ }^{9}$ A recent experimental study using human isolated subcutaneous resistance artery and ventricular trabecular muscle preparations showed that, at test concentrations encompassing free plasma concentrations associated with clinical efficacy for conversion of AF, vernakalant had no significant direct effects on human resistance artery tone or ventricular contractility. ${ }^{53}$ No case of HF worsening with vernakalant treatment has been reported in clinical trials. ${ }^{9}$

The superior efficacy of vernakalant in treating recentonset AF is in great contrast with the suspended clinical trial and halted development of an oral formulation of vernakalant. A recently published short report showed in 51 recent-onset AF patients without structural heart diseases that AF conversion to sinus rhythm is faster and the length of hospital stay is shorter with vernakalant than with flecainide or propafenone. ${ }^{54}$ Although vernakalant is more expensive than amiodarone and flecainide, ${ }^{55}$ this reduction in length of hospital stay should reduce patient costs. In addition, rapid conversion to sinus rhythm may prevent or reverse development of the atrial electrical and structural remodeling associated with $\mathrm{AF}$, potentially reducing disease progression and likelihood of stroke; however, no data are yet available on the long-term effects of vernakalant. Many more studies on the safety and efficacy of vernakalant are required to determine its clinical benefits and therapeutic value.

\section{Conclusion}

The currently available clinical studies have shown that intravenous vernakalant is highly efficacious in acute cardioversion of recent-onset short-duration AF, without any documented vernakalant-related life-threatening TdP arrhythmias. 
The antifibrillatory effect of vernakalant rapidly decreases with the duration of $\mathrm{AF}$ and longer-duration (persistent) $\mathrm{AF}$ rarely responds to the drug. In addition, vernakalant appears less effective for acute conversion of AF in association with $\mathrm{HF}$ and the incidence of serious hypotension and ventricular arrhythmia is higher in this patient population. Future studies are warranted to clarify the efficacy of the drug in AF associated with structural heart diseases and to assess its safety profile in high-risk patients with structural heart disease and left-ventricular dysfunction.

\section{Acknowledgments}

The authors' work was supported by the European Network for Translational Research in Atrial Fibrillation (EUTRAF), the German Federal Ministry of Education and Research (AF Competence Network and the Deutsches Zentrum für HerzKreislauf-Forshcung [German Center for Cardiovascular Research]), the Deutsche Forschungsgemeinschaft (Do 769/1-3), the Fondation Leducq (European-North American Atrial Fibrillation Research Alliance; 07CVD03), the Mochida Memorial Foundation for Medical and Pharmaceutical Research, the Mitsubishi Pharma Research Foundation, the Suzuken Memorial Foundation, and APEX (Nagoya, Japan).

\section{Disclosure}

Dr Dobrev is an advisor and lecturer for Sanofi and Merck-SharpDohme. Dr Tsuji declares no conflicts of interest in this work.

\section{References}

1. Lip GY, Tse HF, Lane DA. Atrial fibrillation. Lancet. 2012;379(9816): 648-661.

2. de Denus S, Sanoski CA, Carlsson J, Opolski G, Spinler SA. Rate vs rhythm control in patients with atrial fibrillation: a meta-analysis. Arch Intern Med. 2005;165(3):258-262.

3. Lafuente-Lafuente C, Mouly S, Longás-Tejero MA, Mahé I, Bergmann JF. Antiarrhythmic drugs for maintaining sinus rhythm after cardioversion of atrial fibrillation: a systematic review of randomized controlled trials. Arch Intern Med. 2006;166(7):719-728.

4. Freemantle N, Lafuente-Lafuente C, Mitchell S, Eckert L, Reynolds M. Mixed treatment comparison of dronedarone, amiodarone, sotalol, flecainide, and propafenone, for the management of atrial fibrillation. Europace. 2011;13(3):329-345.

5. Camm J. Antiarrhythmic drugs for the maintenance of sinus rhythm: risks and benefits. Int J Cardiol. 2012;155(3):362-371.

6. Dobrev D, Nattel S. New antiarrhythmic drugs for treatment of atrial fibrillation. Lancet. 2010;375(9721):1212-1223.

7. Dobrev D, Hamad B, Kirkpatrick P. Vernakalant. Nat Rev Drug Discov. 2010;9(12):915-916.

8. Fedida D, Orth PM, Chen JY, et al. The mechanism of atrial antiarrhythmic action of RSD1235. J Cardiovasc Electrophysiol. 2005;16(11): 1227-1238.

9. European Medicines Agency (EMA). Assessment Report for Brinavess. London: EMA; 2010. Available from: http:/www.ema.europa.eu/ docs/en_GB/document_library/EPAR_-_Public_assessment_report/ human/001215/WC500097150.pdf. Accessed April 3, 2013.
10. Dorian P, Pinter A, Mangat I, Korley V, Cvitkovic SS, Beatch GN. The effect of vernakalant (RSD1235), an investigational antiarrhythmic agent, on atrial electrophysiology in humans. J Cardiovasc Pharmacol. 2007;50(1):35-40.

11. Roy D, Rowe BH, Stiell IG, et al; CRAFT Investigators. A randomized, controlled trial of RSD1235, a novel anti-arrhythmic agent, in the treatment of recent onset atrial fibrillation. J Am Coll Cardiol. 2004; 44(12):2355-2361.

12. Roy D, Pratt CM, Torp-Pedersen C, et al; Atrial Arrhythmia Conversion Trial Investigators. Vernakalant hydrochloride for rapid conversion of atrial fibrillation: a phase 3, randomized, placebo-controlled trial. Circulation. 2008;117(12):1518-1525.

13. Kowey PR, Dorian P, Mitchell LB, et al; Atrial Arrhythmia Conversion Trial Investigators. Vernakalant hydrochloride for the rapid conversion of atrial fibrillation after cardiac surgery: a randomized, doubleblind, placebo-controlled trial. Cir Arrhythm Electrophysiol. 2009; 2(6):652-659.

14. Pratt CM, Roy D, Torp-Pedersen C, et al; Atrial Arrhythmia Conversion Trial (ACT-III) Investigators. Usefulness of vernakalant hydrochloride injection for rapid conversion of atrial fibrillation. Am J Cardiol. 2010;106(9):1277-1283.

15. Stiell IG, Roos JS, Kavanagh KM, Dickinson G. A multicenter, openlabel study of vernakalant for the conversion of atrial fibrillation to sinus rhythm. Am Heart J. 2010;159(6):1095-1101.

16. Camm AJ, Capucci A, Hohnloser SH, et al. A randomized activecontrolled study comparing the efficacy and safety of vernakalant to amiodarone in recent-onset atrial fibrillation. J Am Coll Cardiol. 2011;57(3):313-321.

17. Torp-Pedersen C, Raev DH, Dickinson G, Butterfield NN, Mangal B, Beatch GN. A randomized, placebo-controlled study of vernakalant (oral) for the prevention of atrial fibrillation recurrence after cardioversion. Cir Arrhythm Electrophysiol. 2011;4(5):637-643.

18. Fedida D. Vernakalant (RSD1235): a novel, atrial-selective antifibrillatory agent. Expert Opin Investig Drugs. 2007;16(4):519-532.

19. Wettwer E, Christ T, Endig S, et al. The new antiarrhythmic drug vernakalant: ex vivo study of human atrial tissue from sinus rhythm and chronic atrial fibrillation. Cardiovasc Res. 2013;98(1):145-154.

20. Orth PM, Hesketh JC, Mak CK, et al. RSD1235 blocks late $\mathrm{I}_{\mathrm{Na}}$ and suppresses early afterdepolarizations and torsades de pointes induced by class III agents. Cardiovasc Res. 2006;70(3):486-496.

21. Carmeliet E, Mubagwa K. Antiarrhythmic drugs and cardiac ion channels: mechanisms of action. Prog Biophys Mol Biol. 1998;70(1):1-72.

22. Burashnikov A, Antzelevitch C. New developments in atrial antiarrhythmic drug therapy. Nat Rev Cardiol. 2010;7(3):139-148.

23. Comtois P, Sakabe M, Vigmond EJ, et al. Mechanisms of atrial fibrillation termination by rapidly unbinding $\mathrm{Na}^{+}$channel blockers: insights from mathematical models and experimental correlates. Am J Physiol Heart Circ Physiol. 2008;295(4):H1489-H1504.

24. Antzelevitch C, Burashnikov A. Atrial-selective sodium channel block as a novel strategy for the management of atrial fibrillation. Ann NY Acad Sci. 2010;1188(1):78-86.

25. Burashnikov A, Di Diego JM, Zygmunt AC, Belardinelli L, Antzelevitch C. Atrium-selective sodium channel block as a strategy for suppression of atrial fibrillation: differences in sodium channel inactivation between atria and ventricles and the role of ranolazine. Circulation. 2007;116(13):1449-1457.

26. Burashnikov A, Di Diego JM, Sicouri S, Ferreiro M, Carlsson L, Antzelevitch C. Atrial-selective effects of chronic amiodarone in the management of atrial fibrillation. Heart Rhythm. 2008;5(12):1735-1742.

27. Burashnikov A, Pourrier M, Gibson JK, Lynch JJ, Antzelevitch C. Rate-dependent effects of vernakalant in the isolated non-remodeled canine left atria are primarily due to block of the sodium channel: comparison with ranolazine and dl-sotalol. Cir Arrhythm Electrophysiol. 2012;5(2):400-408.

28. Suzuki T, Morishima M, Kato S, Ueda N, Honjo H, Kamiya K. Atrial selectivity in $\mathrm{Na}^{+}$channel blockade by acute amiodarone. Cardiovasc Res. 2013;98(1):136-144. 
29. Wakili R, Voigt N, Kaab S, Dobrev D, Nattel S. Recent advances in the molecular pathophysiology of atrial fibrillation. J Clin Invest. 2011;121(8):2955-2968.

30. Chen YJ, Chen SA, Chen YC, et al. Effects of rapid atrial pacing on the arrhythmogenic activity of single cardiomyocytes from pulmonary veins: implication in initiation of atrial fibrillation. Circulation. 2001; 104(23):2849-2854.

31. Patterson E, Jackman WM, Beckman KJ, et al. Spontaneous pulmonary vein firing in man: relationship to tachycardia-pause early afterdepolarizations and triggered arrhythmia in canine pulmonary veins in vitro. J Cardiovasc Electrophysiol. 2007;18(10):1067-1075.

32. Voigt N, Li N, Wang Q, et al. Enhanced sarcoplasmic reticulum $\mathrm{Ca}^{2+}$ leak and increased $\mathrm{Na}^{+}-\mathrm{Ca}^{2+}$ exchanger function underlie delayed afterdepolarizations in patients with chronic atrial fibrillation. Circulation. 2012;125(17):2059-2070.

33. Arora R, Verheule S, Scott L, et al. Arrhythmogenic substrate of the pulmonary veins assessed by high-resolution optical mapping. Circulation. 2003;107(13):1816-1821.

34. Kumagai K, Tojo $\mathrm{H}$, Noguchi $\mathrm{H}$, et al. Effects of the $\mathrm{Na}^{+}$channel blocker pilsicainide on the electrophysiologic properties of pulmonary veins in patients with atrial fibrillation. J Cardiovasc Electrophysiol. 2004;15(12):1396-1401.

35. Sicouri S, Pourrier M, Gibson JK, Lynch JJ, Antzelevitch C. Comparison of electrophysiological and antiarrhythmic effects of vernakalant, ranolazine, and sotalol in canine pulmonary vein sleeve preparations. Heart Rhythm. 2012;9(3):422-429.

36. Tian D, Frishman WH. Vernakalant: a new drug to treat patients with acute onset atrial fibrillation. Cardiol Rev. 2011;19(1):41-44.

37. Duggan ST, Scott LJ. Intravenous vernakalant: a review of its use in the management of recent-onset atrial fibrillation. Drugs. 2011; 71(2):237-252.

38. Bronis K, Metaxa S, Koulouris S, Manolis AS. Vernakalant: review of a novel atrial selective antiarrhythmic agent and its place in current treatment of atrial fibrillation. Hospital Chronicles. 2012;7(3):171-181.

39. Blomstrom-Lundqvist C, Blomstrom P. Safety and efficacy of pharmacological cardioversion of atrial fibrillation using intravenous vernakalant, a new antiarrhythmic drug with atrial selectivity. Expert Opin Drug Saf. 2012;11(4):671-679.

40. Cialdella P, Pedicino D, Santangeli P. Novel agents for the acute conversion of atrial fibrillation: focus on vernakalant. Recent Pat Cardiovasc Drug Discov. 2011;6(1):1-8.

41. Vizzardi E, Salghetti F, Bonadei I, et al. A new antiarrythmic drug in the treatment of recent onset atrial fibrillation: vernakalant. Cardiovasc Ther. Epub February 8, 2013.

42. Torp-Pedersen C, Camm AJ, Butterfield NN, Dickinson G, Beatch GN. Vernakalant: Conversion of atrial fibrillation in patients with ischemic heart disease. Int J Cardiol. Epub November 21, 2011.

43. Camm AJ, Toft E, Torp-Pedersen C, et al; Scene 2 Investigators. Efficacy and safety of vernakalant in patients with atrial flutter: a randomized, double-blind, placebo-controlled trial. Europace. 2012;14(6):804-809.

44. de Riva-Silva M, Montero-Cabezas JM, Salgado-Aranda R, López-Gil M, Fontenla-Cerezuela A, Arribas-Ynsaurriaga F. 1:1 atrial flutter after vernakalant administration for atrial fibrillation cardioversion. Rev Esp Cardiol. 2012;65(11):1062-1064. English and Spanish.

45. European Heart Rhythm Association; European Association for CardioThoracic Surgery, Camm AJ, Kirchhof P, et al. Guidelines for the management of atrial fibrillation: the Task Force for the Management of Atrial Fibrillation of the European Society of Cardiology (ESC). Eur Heart J. 2010;31(19):2369-2429.

46. Maastricht University Medical Center. Vernakalant versus ibutilide in recent-onset atrial fibrillation. In: ClinicalTrials.gov [website on the Internet]. Bethseda, MD: US National Library of Medicine; 2012 [updated July 18, 2012]. Available from: http://clinicaltrials.gov/ ct2/show/NCT01646281. NLM identifier: NCT01646281. Accessed April 3, 2013.
47. Medical University of Vienna. Vernakalant versus ibutilide in recentonset atrial fibrillation. In: ClinicalTrials.gov [website on the Internet]. Bethseda, MD: US National Library of Medicine; 2011 [updated February 21, 2012]. Available from: http://clinicaltrials.gov/ct2/show/ NCT01447862. NLM identifier: NCT01447862. Accessed April 3, 2013.

48. Bash LD, Buono JL, Davies GM, et al. Systematic review and metaanalysis of the efficacy of cardioversion by vernakalant and comparators in patients with atrial fibrillation. Cardiovasc Drugs Ther. 2012;26(2):167-179.

49. Fuster V, Rydén LE, Cannom DS, et al; American College of Cardiology/American Heart Association Task Force on Practice Guidelines; European Society of Cardiology Committee for Practice Guidelines; European Heart Rhythm Association; Heart Rhythm Society. ACC/AHA/ESC 2006 Guidelines for the Management of Patients with Atrial Fibrillation: a report of the American College of Cardiology/American Heart Association Task Force on Practice Guidelines and the European Society of Cardiology Committee for Practice Guidelines (Writing Committee to Revise the 2001 Guidelines for the Management of Patients With Atrial Fibrillation): developed in collaboration with the European Heart Rhythm Association and the Heart Rhythm Society. Circulation. 2006;114(7):e257-e354.

50. Duytschaever M, Blaauw Y, Allessie M. Consequences of atrial electrical remodeling for the anti-arrhythmic action of class IC and class III drugs. Cardiovasc Res. 2005;67(1):69-76.

51. Eijsbouts S, Ausma J, Blaauw Y, Schotten U, Duytschaever M, Allessie MA. Serial cardioversion by class IC Drugs during 4 months of persistent atrial fibrillation in the goat. J Cardiovasc Electrophysiol. 2006;17(6):648-654.

52. Buccelletti F, Iacomini P, Botta G, et al. Efficacy and safety of vernakalant in recent-onset atrial fibrillation after the European Medicines Agency approval: systematic review and meta-analysis. J Clin Pharmacol. 2012;52(12):1872-1878.

53. Lynch JJ Jr, Regan CP, Beatch GN, Gleim GW, Morabito CJ. Comparison of the intrinsic vasorelaxant and inotropic effects of the antiarrhythmic agents vernakalant and flecainide in human isolated vascular and cardiac tissues. J Cardiovasc Pharmacol. 2013;61(3):226-232.

54. Conde D, Costabel JP, Aragon M, et al. Flecainide or propafenone vs vernakalant for conversion of recent-onset atrial fibrillation. Can J Cardiol. Epub March 2, 2013.

55. Andresen D, Trappe HJ. Antiarrhythmic drug therapy in patients with supraventricular or ventricular tachyarrhythmias in emergencies. Appl Cardiopulm Pathophysiol. 2012;16(2):154-161.

56. Kodama I, Kamiya K, Toyama J. Amiodarone: ionic and cellular mechanisms of action of the most promising class III agent. Am J Cardiol. 1999;84(9):20-28.

57. Gautier P, Guillemare E, Djandjighian L, et al. In vivo and in vitro characterization of the novel antiarrhythmic agent SSR149744C: electrophysiological, anti-adrenergic, and anti-angiotensin II effects. J Cardiovasc Pharmacol. 2004;44(2):244-257.

58. Aliot E, Capucci A, Crijns HJ, Goette A, Tamargo J. Twenty-five years in the making: flecainide is safe and effective for the management of atrial fibrillation. Europace. 2011;13(2):161-173.

59. Voigt N, Rozmaritsa N, Trausch A, et al. Inhibition of $I_{K, A C h}$ current may contribute to clinical efficacy of class I and class III antiarrhythmic drugs in patients with atrial fibrillation. Naunyn Schmiedebergs Arch Pharmacol. 2010;381(3):251-259. 


\section{Publish your work in this journal}

Vascular Health and Risk Management is an international, peerreviewed journal of therapeutics and risk management, focusing on concise rapid reporting of clinical studies on the processes involved in the maintenance of vascular health; the monitoring, prevention and treatment of vascular disease and its sequelae; and the involvement of metabolic disorders, particularly diabetes. This journal is indexed on PubMed Central and MedLine. The manuscript management system is completely online and includes a very quick and fair peer-review system, which is all easy to use. Visit http://www.dovepress.com/ testimonials.php to read real quotes from published authors.

Submit your manuscript here: http://www.dovepress.com/vascular-health-and-risk-management-journal 